Annuaire suisse de politique de développement

26-2 | 2007

Financer le développement par la mobilisation des ressources locales

\title{
La microassurance : retour sur un besoin essentiel
}

Marc Nabeth

\section{OpenEdition}

Journals

Édition électronique

URL : http://journals.openedition.org/aspd/136

ISSN : 1663-9669

\section{Éditeur}

Institut de hautes études internationales et du développement

Édition imprimée

Date de publication : 1 novembre 2007

Pagination : 125-142

ISBN : 978-2-88247-068-3

ISSN : $1660-5934$

\section{Référence électronique}

Marc Nabeth, «La microassurance : retour sur un besoin essentiel », Annuaire suisse de politique de développement [En ligne], 26-2 | 2007, mis en ligne le 22 juin 2009, consulté le 07 septembre 2020. URL : http://journals.openedition.org/aspd/136 


\title{
La microassurance:
}

\section{retour sur un besoin essentiel}

\author{
Marc Nabeth*
}

D

ans ce qui relie pauvreté et vulnérabilité, la fluctuation des revenus est une donnée essentielle. Accentuant un peu plus les vulnérabilités, cette fluctuation entretient la pauvreté ou la provoque dès lors que des sinistres difficilement prévisibles (décès d'un membre de la famille, problèmes de santé, dommages aux biens, etc.) viennent grever les revenus d'un ménage: «D'un point de vue matériel, être pauvre est moins le fait d'avoir des ressources très faibles par rapport au système de besoins prévalant dans sa société que le fait d'une perception erratique des revenus », souligne Jean-Michel Servet ${ }^{1}$.

C'est en atténuant les fluctuations de revenus causées par des sinistres que la microassurance (assurance adaptée aux populations à faible revenu) participe à la réduction des pauvretés et des incertitudes. Le mécanisme est connu: moyennant le paiement préalable d'une prime (ou cotisation), l'assuré reçoit de l'assureur une prestation (indemnisation financière ou en nature) en cas de réalisation du sinistre prévu dans le contrat. Cette réduction des incertitudes n'est pas négligeable, pour des populations particulièrement vulnérables: elle peut constituer l'ultime filet de sécurité contre le risque d'indigence et contribuer à l'ouverture d'autres possibles en proposant une relative tranquillité d'esprit.

L'assurance est pourtant souvent considérée comme un luxe, un instrument secondaire difficilement compatible avec la notion de pauvreté. Cette réduction de l'assurance aux seules catégories privilégiées (pays riches et nantis des pays en développement) semble confirmée par quelques statistiques mondiales, telles que la répartition des cotisations mondiales, les primes encaissées, le taux de pénétration de l'assurance ou la densité de l'assurance (voir tableau).

Tableau: Quelques chiffres sur l'assurance dans le monde, 2005

\begin{tabular}{|c|c|c|}
\hline 2005 & Pays industrialisés & $\begin{array}{l}\text { Pays émergents (Asie, Amérique } \\
\text { latine, Afrique, Europe orientale, } \\
\text { Moyen-Orient) }\end{array}$ \\
\hline Répartition des cotisations mondiales & $88 \%$ & $12 \%$ \\
\hline Primes & $\begin{array}{l}1717 \text { milliards de dollars (vie) } \\
1281 \text { milliards de dollars (non-vie) }\end{array}$ & $\begin{array}{l}256 \text { milliards de dollars (vie) }{ }^{b} \\
171 \text { milliards de dollars (non-vie) }\end{array}$ \\
\hline $\begin{array}{l}\text { Taux de pénétration de l'assurance } \\
\text { (primes directes en \% du PIB) }\end{array}$ & $\begin{array}{l}5,1 \%(\text { vie) } \\
3,8 \% \text { (non-vie) }^{b}\end{array}$ & $\begin{array}{l}2,2 \%{(\text { vie })^{b}}_{1,4 \%(\text { non-vie) }}^{b}\end{array}$ \\
\hline Densité (primes/habitant) & $\begin{array}{l}1887 \text { dollars (vie) } \\
1400 \text { dollars (non-vie) }\end{array}$ & $\begin{array}{l}46 \text { dollars (vie) } \\
31 \text { dollard (non-vie) }\end{array}$ \\
\hline \multicolumn{3}{|c|}{$\begin{array}{l}\text { Source: Swiss Re, L'assurance dans le monde en 2005: croissance modérée des } \\
\text { a II existe de grandes différences entre pays émergents et au sein même de ces } \\
\text { b Sur la distinction entre assurance vie et assurance non-vie, voir l'encadré } 1 .\end{array}$} \\
\hline $\begin{array}{l}\text { * Consultant à CGSI-consulting } \\
\text { (Paris-Bruxelles). } \\
1 \text { J.-M. Servet, Banquiers aux pie }\end{array}$ & Suresnes, France) et chercheur & associé à l'Institut Thomas More \\
\hline
\end{tabular}




\section{Encadré 1: Distinction entre assurance vie et assurance non-vie}

\section{Assurance vie}

\section{Assurances en cas de décès}

Exemple: la " temporaire décès" garantit un capital ou une rente en cas de décès de la personne assurée avant le terme du contrat Assurances en cas de vie

Exemple: le capital différé assure un capital qui est payé au terme du contrat à la condition que l'assuré soit en vie à cette date. Cela s'apparente à de l'épargne revalorisée Assurances mixtes

Ce contrat est l'alliance d'une temporaire décès et d'un capital différé. A titre d'exemple, un mixte 20/80 prévoira pour le(s) bénéficiaire(s) ${ }^{a}$ un capital de 20 en cas de décès avant le terme du contrat. Si l'assuré est en vie au terme, le capital de 80 est alors payé à l'assuré.
Assurance non-vie

Assurances de biens

Exemples: vol, incendie

Assurances de responsabilité

Elles garantissent les dommages (corporels ou à leurs biens) que l'assuré peut occasionner à des tiers

Assurances de dommages corporels

Exemples: santé, hospitalisation, individuelle accident, etc.

a Bénéficiaire: personne qui perçoit la prestation d'assurance en cas de réalisation du sinistre assuré.

Le tableau de Swiss Re (voir page précédente) distingue l'assurance vie de l'assurance non-vie. Cette distinction est peu usitée parmi les assureurs français ou francophones qui préfèrent distinguer entre "assurances de personnes" et "assurances de biens et responsabilité ».

Les assurances de personnes ayant pour objet le versement de prestations en cas d'évènement affectant la personne même de l'assuré (décès, accident, maladie, incapacité-invalidité); les assurances de biens et responsabilités ayant pour objectif de réparer les conséquences d'un événement dommageable affectant le patrimoine de l'assuré (vol, incendie, perte d'exploitation d'un outil de production, etc.).

Pour autant, des réflexions sur un avenir peut-être proche et quelques retours sur un passé pas si lointain invitent à dépasser une forme de tyrannie des chiffres et du présent. Ainsi Philippe Trainar, chef économiste à $\mathrm{SCOR}^{2}$, s'interroge sur l'émergence d'une microassurance, dans le sillage de la microfinance, qui pourrait bien remettre en cause le lieu commun d'une assurance comme bien supérieur $^{3}$, ou Craig Churchill, président du groupe de travail CGAP (Groupe consultatif d'assistance aux pauvres) sur la microassurance, constate: «Les assureurs supposent, à tort ou à raison, que les populations à faible revenu n'ont pas les moyens de s'assurer. Intéressant, quand on sait que lorsque l'assurance s'est diffusée à la fin du XIX ${ }^{\mathrm{e}}$ siècle, elle était vue comme un service financier pour les pauvres. Les riches n'avaient pas besoin de s'assurer puisqu'ils pouvaient s'autoassurer. $»^{4}$

La microassurance n'est cependant pas qu'une leçon d'histoire. Elle a bien plus à nous apprendre sur le présent. «Ce que la microassurance fait ressortir, c'est l'incapacité des couvertures conçues pour les pays développés ou pour la frange

2 SCOR est le premier réassureur français.

3 P. Trainar, «Préface», in M. Nabeth, Micro-assurance. Défis, mise en place et commercialisation, Les Fondamentaux de l'assurance, Paris, Dalloz; L'Argus de l'Assurance, 2006.

4 C. Churchill, «What Is Insurance for the Poor?», in C.F. Churchill (ed.), Protecting the Poor: A Microinsurance Compendium, Munich, Munich Re Foundation; Geneva, ILO, 2006, p. 20. 
fortunée des pays en développement à fournir une solution pour la frange pauvre de ces pays », note à juste titre Philippe Trainar ${ }^{5}$. Nous serions tentés de dire que cela pourrait aussi s'appliquer à nos nouvelles formes de sociétés postindustrielles, confrontées à la crise du salariat, à l'éclatement des inégalités au sein de chaque groupe socioculturel et à l'évolution chaotique du revenu des individus eux-mêmes au cours de leur vie ${ }^{6}$.

\section{La microassurance n'a pas réponse à tout}

Au-delà de la problématique des vulnérabilités, la microassurance soulève la question de l'Etat de droit et de l'éthique d'une société. En effet, la microassurance est bien incapable de réduire les vulnérabilités dans des zones de conflit armé ou écrasées par un pouvoir totalitaire. La microassurance ne résoudra pas plus que le microcrédit la pauvreté massive au Darfour ou en Corée du Nord. La question éthique renvoie quant à elle à celle des mutualisations assumées ou partagées par l'Etat et les opérateurs économiques. Il ne s'agit certes pas de confondre fonction assurantielle et principe de redistribution sociale, mais plutôt d'appréhender les périmètres de mutualisation au sein d'une société. Un périmètre vide d'un pan entier d'une société (cas de pays en développement) ou dépourvu de sens pour la majorité des citoyens (cas des pays industrialisés) ne serait-il pas le signe d'une société en immaturité démocratique?

Ces questions se retrouvent d'une certaine façon dans la polysémie de la microassurance, même si l'objectif commun de tous ses promoteurs reste in fine la réduction des vulnérabilités de populations à faible revenu.

\section{Pauvreté et microassurance: des définitions multiples}

\section{Diverses pauvretés}

La pauvreté n'est pas d'un bloc. Résultante d'un processus socio-économique complexe, sur fond d'exclusion des mécanismes de redistribution (indigence) ou de différenciations sociales (sexe, âge, ethnie, profession principale ou caste, ancienneté d'installation, parfois religion, niveau de richesse...), la pauvreté est un phénomène dynamique qui regroupe des multitudes de trajectoires et de situations ${ }^{7}$. Les «très pauvres», les «pauvres», les «moyens pauvres», les «nonpauvres » s'apparentent à des classes d'objets abstraites alors qu'il s'agirait plutôt d'analyser les continuums au lieu d'établir des coupures radicales. La compréhension par un microassureur des représentations objectives (ce qui s'observe aisément) et subjectives (ce qui est exprimé) de la pauvreté chez les populations

P. Trainar, ibid., p. 13.

6 D. Cohen, Richesse du monde, pauvretés des nations, Paris, Flammarion, 1997. Les phénomènes de pauvretés plus ou moins massives, de pression démographique considérable, d'absence de protection sociale pour la très grande majorité des populations des pays en développement rendent cependant la situation encore bien plus critique dans ces pays.

7 E. Baumann, «Microentreprise et gestion de la vulnérabilité en Afrique subsaharienne, passé et présent», ADA Dialogue, no 33, 2004, pp. 31-53. D. Gentil, «Acteurs, normes et formes de régulation dans la microfinance», in M. Lévy (dir.), Comment réduire pauvreté et inégalités. Pour une méthodologie des politiques publiques, Paris, IRD; Karthala, 2002, pp. 97-124. 
est un préalable. Elle permet notamment de définir la meilleure stratégie à adopter, en tenant compte du contexte socioculturel, des rapports entre l'individu et la collectivité et, finalement, des gestions du risque jusqu'alors déployées.

Ainsi, au Mali, le mot «pauvreté» se définit par l'impuissance, fangantan (en langue bamanan), par opposition à la puissance, fangama, qui permet certes d'accéder à la richesse mais oblige en contrepartie le «puissant » à redistribuer ses ressources considérées comme un bien public. Cela afin de gagner le prestige privé qui est attaché à la détention nécessairement temporaire du pouvoir ${ }^{8}$. La conséquence, en termes de mutualisation des risques et d'inclusion des plus pauvres dans ce système, sera en revanche bien différente de celle résultant des pratiques en pays Bamiléké (Cameroun). En effet, en pays Bamiléké, le pauvre est un «non-chanceux», un balock soupçonné par ailleurs de ne pas être sérieux et donc à éviter: tous les pauvres sont potentiellement soupçonnés de «désaccumuler», d'être des paniers percés, et sont menacés comme tels d'être mis hors solidarité ${ }^{\text {. }}$.

De cette diversité des situations découle probablement la difficulté à rassembler tous les acteurs autour d'une définition consensuelle des «populations à faible revenu » comme de la microassurance. Le draft de l'International Association of Insurance Supervisors (IAIS), issu d'une réunion consacrée à la microassurance en 2006, illustre ainsi ces difficultés. Revenant dans un premier temps sur la définition de la microassurance, qui «ne porte pas sur des produits ou lignes de produits spécifiques, n'est pas limitée à un type de producteurs, mais se réfère à une offre à destination d'un certain niveau de revenu; à savoir les populations à faible revenu», l'IAIS précise dans un second temps et à plusieurs reprises que non seulement le concept de «populations à faible revenu » varie d'un pays à l'autre, en fonction de l'état de son développement, mais encore que la microassurance a des significations différentes selon les autorités de contrôle de l'assurance. "Dans beaucoup de pays, la microassurance n'est pas considérée comme une assurance à part, mais simplement comme une assurance classique à petite échelle. Ce qui est l'une des explications du non-développement de règles séparées pour la microassurance dans beaucoup de juridictions. ${ }^{10}$

\section{Exemples des différentes définitions de la microassurance}

La diversité des situations et des définitions n'est pas sans impact sur les fonctions et les objectifs de la microassurance. Doit-elle ainsi relever de la protection sociale ou de l'assurance commerciale? Faut-il inclure les indigents ou plutôt les classes moyennes dépourvues de protection sociale et assurantielle? Doit-elle viser uniquement le secteur informel ou s'intéresser également aux working poor salariés? Doit-elle rester dans le giron de l'Etat, s'inscrire dans le cadre du marché, se définir comme une nouvelle forme de politique de subsidiarité des Etats subventionnant en partie les programmes assurantiels des acteurs

8 H. Magassa, «Ethique et pauvreté: l'exemple du Mali», in collectif, La pauvreté, une fatalité ?, coll. Tropiques, Paris, Karthala, 2002, pp. 129-176.

9 J.-P. Warnier, L'esprit d'entreprise au Cameroun, Paris, Karthala, 1993.

10 International Association of Insurance Supervisors and CGAP Working Group on Microinsurance, Issues in Regulation and Supervision of Microinsurance, November 2006. 
économiques et sociaux, locaux ou internationaux ? Force est de constater que la microassurance prend un sens suffisamment large pour qu'on la retrouve simultanément sous différents termes dans des articles, des communications ou des textes juridiques.

En Afrique australe, le terme de low cost est ainsi aisément employé pour définir l'assurance des populations à faible revenu ${ }^{11}$. En Afrique du Sud, cette assurance low cost cible principalement les ménages rangés dans les catégories LSM (living standards measures) de 1 (la plus pauvre) à 5, soit 17,5 millions d'adultes environ, dont 9,5 millions gagnant moins de 500 rands par mois.

En Inde, l'Indian Regulatory and Development Authority (IRDA) associe pour sa part la microassurance à des produits vie et non-vie pour des catégories à faible revenu du secteur social et du secteur rural. Imitant en quelque sorte les politiques volontaristes de lutte contre les discriminations, cette instance de réglementation impose des quotas conséquents aux assureurs privés ${ }^{12}$ (voir encadré 2).

Au Brésil, au-delà d'une distinction sémantique subtile, l'assurance populaire s'apparente ou reste très proche de la microassurance, comme en témoignent les produits populaires proposés («temporaires décès» de la compagnie d'assurances SINAF à partir de 3.50 euros par mois pour une garantie de 1000 euros, une participation aux frais d'obsèques et une assistance alimentaire ${ }^{13}$ ), les partenariats de distribution noués (Casas Bahia, un détaillant en produits domestiques et ménagers, avec le bancassureur Bradesco), les classes moyennes basses ciblées $(\mathrm{C} \text { et } \mathrm{D})^{14}$ et des interventions publiques de la Superintendence des assurances privées (SUSEP), l'instance de régulation et de contrôle des assureurs privés au Brésil (voir encadré 2) ${ }^{15}$.

En France, la première initiative de microassurance développée par la fondation Entrepreneurs de la Cité est qualifiée de solidaire, collective et universelle. Cette fondation, constituée autour d'un pool d'assureurs (AG2R, April Group, CNP Assurances, La Mondiale), d'établissements financiers (La Banque Postale, La Caisse des Dépôts, EVOLEM) et de réseaux d'aide à la création d'entreprise (Association pour le droit à l'initiative économique ADIE, France active, France initiative, Réseau des boutiques de gestion), ne fait en effet payer aux créateurs d'entreprises (anciens Rmistes ou chômeurs) que la prime de risque, les frais de

11 Voir notamment les articles de FinMark Trust et d'Atlas Magazine. FinMark Trust est une société fiduciaire indépendante contrôlée par des administrateurs émanant des pays sud-africains (<http://www.finmarktrust.org.za>). Atlas Magazine est la revue d'Atlas Conseil International, organisation indépendante basée à Tunis, spécialisée dans l'assurance et la réassurance des pays émergents (<http://www.atlasconseil.com.tn>).

12 M. Nabeth, «Réduire la vulnérabilité des populations "pauvres" par la microassurance: une synergie entre les Etats et les industriels de l'assurance», Risques, no 65, mars 2006, pp. 110-114.

13 Résultat: 300000 polices vendues par cette banque en moins de deux ans.

14 On distingue au Brésil sept classes sociales, en fonction du revenu moyen mensuel d'un ménage. La classe A1 et A2, soit 5,1\% de la population, gagne plus de 6630 dollars par mois. Au «bas de la pyramide», nous trouvons la classe E, soit $2,7 \%$ de la population, gagnant moins de 300 dollars par mois. Les classes $\mathrm{C}$ et $\mathrm{D}$, juste au-dessus, regroupent les ménages gagnant plus de 570 dollars par mois et 1140 dollars par mois, soit $28,8 \%$ et $39,3 \%$ de la population.

15 Moacir Lamha Filho, procureur général de la SUSEP, «Inclusion sociale de l'assurance», $3^{\mathrm{e}}$ Forum international du Centre des hautes études d'assurances, Paris, CHEA, 5 décembre 2006. E. Lorenzon, «Le Brésil: futur grand marché de l'assurance?», conférence, Paris, CHEA, 5 décembre 2006. 
gestion et de commercialisation étant à la charge de la fondation ${ }^{16}$. Mais déjà se dessinent à l'horizon d'autres formes de microassurance, avec pour objectif de proposer également des garanties moins onéreuses et plus adaptées aux spécificités des microentreprises ( $57 \%$ des entreprises en France), des très petites entreprises (TPE, 39\%) ou des particuliers en situation de plus grande vulnérabilité.

\section{Encadré 2: Le rôle moteur de certaines instances de régulation}

L'Indian Regulatory and Development Authority (IRDA) et la Superintendence brésilienne des assurances privées (SUSEP) sont particulièrement actives dans la promotion de la microassurance. La première privilégie la voie des contraintes réglementaires et la seconde, celle de la défiscalisation.

\section{L'Inde}

Le premier document juridique, qui date de 2002, est intitulé Obligations of Insurers to Rural Social Sectors. II exige des assureurs privés la vente d'un certain pourcentage de contrats d'assurance à des clients "à faible revenu», selon des quotas évolutifs. Dès la cinquième année d'activité, les assureurs vie doivent consacrer au secteur rural au moins $16 \%$ du total des contrats émis (population de moins de 5000 âmes, densité de population inférieure à 400 hab/ $/ \mathrm{km}^{2}$, plus de $25 \%$ des hommes engagés dans des activités agricoles), et couvrir 20000 personnes du secteur social (incluant le secteur informel, le secteur non organisé et les classes rurales ou urbaines dites vulnérables). Les assureurs non-vie doivent pour leur part consacrer au secteur rural au moins $5 \%$ du total des cotisations brutes collectées et couvrir 20000 personnes du secteur social.

Un texte plus récent, intitulé IRDA (Microinsurance) Regulations 2005, énonce différentes règles liées à I'activité de microassurance, dont les caractéristiques des agents de distribution de microassurance (commissionnement, règles de conduite, etc.) et des produits de microassurance (types de garantie, sommes maximale et minimale de couverture, durées de garantie, âges limites de souscription, etc.).

De nombreux débats existent cependant sur l'opportunité, la clarté et l'efficacité de ces réglementations.

\section{Le Brésil}

Pour la SUSEP, la microassurance est devenue un secteur essentiel en raison de son rôle d'inclusion sociale.

La SUSEP facilite, notamment à travers la circulaire Circular SUSEP n 267/2004, les conditions d'accès des populations à faible revenu à l'assurance vie, en levant notamment les obstacles liés aux réseaux de distribution (banques de détail et populaires, Eglises, etc.). Elle a ainsi permis aux prêtres de distribuer, en même temps qu'une Bible, des contrats d'assurance.

A travers une seconde circulaire, Circular SUSEP n ${ }^{\circ} 306 / 2005$, la SUSEP vise à promouvoir l'assurance du parc automobile des voitures d'occasion (véhicules de dix à vingt ans d'usage), celles-ci étant largement utilisées par les classes populaires brésiliennes.

La SUSEP souhaite également promouvoir l'assurance rurale.

16 La fondation propose une garantie prévoyance et multi-risque professionnelle pour 21.92 euros par mois, hors complémentaire santé (17.75 euros pour un adulte seul) et pour une durée maximale de quatre ans, la microassurance étant «un tremplin vers les produits ordinaires». La cotisation correspond au coût technique sans chargement. 
La microassurance est donc bel et bien plurielle. Elle s'incarne aussi bien dans les mutuelles de santé soutenues par des bailleurs de fonds et des ONG que dans des programmes développés par de grands assureurs capitalistiques. Visant dans certains cas les pauvres parmi les pauvres $(1$ dollar par jour à parité de pouvoir d'achat), en incluant parfois les indigents, la microassurance s'étend dans d'autres cas aux ménages de la classe moyenne à venir ou émergente, toujours fragilisée par l'absence de protection sociale ou d'assurance privée. Ajoutée parfois comme complément aux services de crédit ou d'épargne de certaines institutions de microfinance (IMF), la microassurance va d'autres fois bien audelà du spectre de la microfinance pour devenir une nouvelle forme d'assurance populaire (voir plus haut). Dans de nombreux pays où la protection sociale est massivement déficiente et où les assurances privées ne concernent que $5 \%$ à $15 \%$ de la population du pays (par exemple Brésil, Inde ou Chine!), cela ne saurait surprendre. La microassurance accompagne d'ailleurs une économie populaire en expansion depuis vingt ans, dans un contexte de retrait, recherché ou subi, de l'Etat. A titre d'exemple, et anticipant quelque peu sur nos propos sur le rôle des réseaux de distribution, citons les partenariats entre des associations de femmes et des assureurs commerciaux en Inde (Self Employed Women's Association [SEWA] avec AVIVA) ou en Colombie (l'IMF Women's World Foundation [WWF] avec l'assureur La Equidad) ${ }^{17}$.

\section{Indigence, segmentation ${ }^{18}$, mutualisation}

Alors que certaines ONG jugent essentielle l'inclusion des indigents dans les services de microassurance, quitte à inventer des programmes ingénieux (cas du groupe associatif français CIDR [Centre de développement international et de recherche] avec la création d'une mutuelle de santé «sécurité sociale villageoise » (SSV) à Mnoungou, aux Comores), les assureurs commerciaux jugeront dans la majorité des cas que leur microassurance n'a pas pour vocation de se substituer à une protection sociale. L'indigence, produit d'une exclusion sociale, relèverait bien plus d'une responsabilité de l'Etat ou des communautés que d'un acteur du marché. Il ne s'agit certes pas de stigmatiser les indigents, et de rejoindre dans un certain délire des promoteurs d'une microfinance distinguant les «bons pauvres» (les catégories sociales à faible capital initial mais ayant des projets à financer, tels que les jeunes, les apprentis, les veuves, les microentrepreneurs...) et le «bon crédit» (crédit d'investissement) des «mauvais pauvres» (ceux qui ne voudraient pas prendre de risques, tels que les personnes âgées ou tous ceux qui restent soumis à diverses formes de dépendance) et du «mauvais crédit» (crédit à la consommation $)^{19}$. Il s'agit plutôt de souligner que la microassurance peut aider à éviter

17 Les agents de WWF proposent à leurs clientes un produit d'assurance de La Equidad garantissant le versement de prestations financières en cas de décès de l'emprunteur ou d'invalidité permanente et irréversible due à un accident. Les frais d'obsèques sont également garantis.

18 La segmentation consiste à répartir les risques dans plusieurs classes présentant chacune une certaine homogénéité des niveaux de risques qu'elles regroupent et dotées du tarif correspondant.

19 On lira avec intérêt la section «L'économie est une négociation linguistique», in B. Maris, L'antimanuel d'économie, t. 1, Rosny-sous-Bois (France), Bréal, 2003. 
l'indigence, en aucun cas à en sortir. Ces nouvelles formes d'assurances soutiennent et encouragent le développement économique et social à travers une prise de risque et des investissements supplémentaires, sans exclure pour autant du champ de la microassurance les non-microentrepreneurs.

La microassurance, de par son principe de mutualisation et de loi des grands nombres ${ }^{20}$, se différencie en ce sens considérablement des visions dogmatiques associant le microcrédit à la seule microentreprise. La connaissance des différents segments de population permet moins d'exclure que d'équilibrer ses portefeuilles, et d'arbitrer entre la nécessité d'exclure des «mauvais risques» et celle d'intégrer ces derniers dans ses portefeuilles. Doit-on en effet exclure un sage du village ou un responsable de communauté en raison d'un âge avancé, ou s'appuyer sur lui pour promouvoir la microassurance? Faut-il, à l'instar d'American International Group (AIG) en Ouganda, supprimer les clauses d'exclusion du sida, après avoir constaté que le refus des personnes séropositives était finalement bien plus préjudiciable au déploiement de l'assurance que leur acceptation ?21

Ce sont autant les raisons éthiques que les problèmes techniques qui expliquent la relative souplesse des assureurs dans leur sélection des risques et leur segmentation, résume Jean-Marc Cazenave, avant de remarquer que la segmentation est un exercice coûteux (choix des critères, estimation des primes, modélisation actuarielle, réactualisation) et parfois risqué (risque d'erreur conséquente si le périmètre du segment n'est pas assez large $)^{22}$.

\section{Deux impératifs: répondre aux demandes et besoins et proposer des produits compréhensibles}

La définition d'un périmètre de mutualisation acceptable tant par les populations que par les assureurs passe nécessairement par l'analyse des structurations sociales, économiques, voire politiques, légitimées par les populations. Humilité des approches qui impose aux assureurs de s'appuyer sur les réseaux traditionnels de solidarité, sur ceux de l'économie populaire, sur ceux en émergence, allant dès lors bien au-delà du cercle des microentreprises et TPE plus ou moins informelles. En s'appuyant sur des réseaux au fait des problématiques des populations à faible revenu (IMF, self-help groups, ONG, chaînes de vente de détail, fonds funéraires semi-formels comme la Great North Burial Society par exemple en Afrique du Sud), en communiquant souvent à travers un langage populaire faisant sens pour les populations, la microassurance incarne ce mélange de tradition et d'innovation essentiel au développement d'un nouveau secteur ${ }^{23}$.

20 Loi des grands nombres: sous l'hypothèse d'indépendance des risques et dans une population d'assurés homogènes, l'indemnité moyenne par assuré tend alors vers l'espérance mathématique de l'indemnité (prime pure) lorsque le nombre d'assurés s'accroît.

${ }_{21}$ M. Nabeth, Micro-assurance. Défis, mise en place et commercialisation, Les Fondamentaux de l'assurance, Paris, Dalloz; L'Argus de l'Assurance, 2006.

22 J.-M. Cazenave, «Les assureurs santé face aux changements d'accès aux données de santé et gestion du risque: une nouvelle donne pour le modèle économique de l'assurance santé ?», thèse MBA, France, Ecole nationale d'assurances (ENAss), 2005.

23 J.-M. Servet, op. cit. 
Cette synthèse n'est réalisable qu'en tenant compte des besoins et des demandes exprimés par les populations, de sorte que l'assureur essaiera de concilier deux nécessités :

$\square$ celle de proposer un produit en adéquation avec les attentes et les besoins des populations. Les demandes pourront différer en fonction de facteurs culturels, socio-économiques, géographiques, de genre, etc ${ }^{24}$;

$\checkmark$ celle de commencer par des produits relativement simples afin de mieux appréhender le secteur de la microassurance. Ces produits seront cependant différents selon l'expertise et l'objectif de l'assureur.

\section{Première nécessité: analyse des besoins et pédagogie de l'assurance comme préalable à la construction de la confiance}

Tout développement de l'assurance et de la microassurance est tributaire de la confiance et de la perception d'une valeur ajoutée par les populations, des plus vulnérables aux classes moyennes basses ou émergentes. Cette confiance dans l'assurance et l'assureur n'est pourtant jamais acquise ${ }^{25}$.

Un changement de paradigme de l'assurance ou, du moins, la prise de conscience du problème de l' «assurabilité» est dès lors requis: «Le problème n'est pas tant dans le manque de capacité, de revenu disponible ou de culture assurantielle de la population que dans la faculté des assureurs à adapter leurs moyens», explique Alfredo Honsberg, directeur général de Seguros Azteca, annonçant le déploiement de programmes de microassurance au Mexique, puis dans différents pays d'Amérique latine ${ }^{26}$.

Ce modèle économique à développer passe notamment par la compréhension des cultures sociétales, ou plutôt des systèmes sociaux, pour reprendre la formule de Christophe Jaffrelot: «Il faut se méfier des explications culturalistes; le réflexe premier est souvent le plus simpliste. En revanche, il existe dans la longue durée des histoires sociales certaines constances qui peuvent être significatives. $»^{27}$

La microassurance s'inscrit d'ailleurs dans un processus d'accompagnement et d'extension des pratiques populaires. Des étudiants de l'école polytechnique remarquent à juste titre, dans leur travail de recherche, qu'au-delà d'une question de «rentabilité» de l'activité d'assureur dans des milieux défavorisés, le

24 Voir notamment les différents travaux de Michal Matul (p. ex. Understanding Demand for Microinsurance in Georgia [contribution au KfW Microinsurance Symposium à Francfort, les 21 et 22 octobre 2004], Warsaw, Microfinance Center, 2004) ainsi que M. Cohen and J. Sebstad, «The Demand for Microinsurance», in C. Churchill (ed.), Protecting the Poor: A Microinsurance Compendium, Munich, Munich Re Foundation; Geneva, ILO, 2006.

25 Voir notamment les travaux de Viviana Zelizer sur la réticence des classes populaires américaines à l'égard de l'assurance vie, p. ex. Morals and Markets: The Development of Life Insurance in the United States, New York, Columbia University Press, 1979

26 «Insurers aim at "microinsurance" market», The Herald (Mexico Edition), 15 de diciembre de 2006. Seguros Azteca suit en ce sens l'exemple de Banamex, une filiale d'assurance mexicaine de la Citigroup.

27 C. Jaffrelot, «Il n'y a pas de miracle», interview de François Godement et Christophe Jaffrelot, in P. Gauchont (dir.), Inde, Chine, à l'assaut du monde: faut-il en avoir peur? Rapport Antheios 2006, Paris, Presses universitaires de France (PUF), 2006. 
véritable défi de la microassurance serait l'insertion d'un système d'assurance de type classique dans un cadre d'assurance informelle. Non pour le cannibaliser, mais presque pour s'en inspirer, et étendre alors les périmètres de mutualisation permettant une meilleure efficacité du système assurantiel ${ }^{28}$.

L'écoute des populations et leur implication dans la définition des produits ainsi que la légitimité des promoteurs de la microassurance, de la direction au réseau de distribution, auprès des populations conditionnent finalement toute approche. Tata AIG, compagnie d'assurance vie indienne, avance plus sur le nom «Tata» (important groupe industriel indien) que sur celui de «AIG», le premier étant bien plus connu que le second, pour créer de la confiance. Les microagents formés par Tata AIG, après avoir été généralement recommandés par des ONG, sont en outre issus du terrain.

Les supports de communication sont également essentiels - à condition d'être signifiants pour les populations. Théâtres ambulants, cinémas, peintures murales, brochures en bande dessinée... les exemples sont nombreux et finalement nullement spécifiques des pays en développement. Jean-François Estienne, spécialiste de l'assurance au Brésil et au Japon, évoque les mangas éducatifs développés par les assureurs japonais ${ }^{29}$. Les assureurs utilisent ici comme ailleurs des messages particulièrement anxiogènes, basés sur la solidarité («Tous pour un, un pour tous» pour Yeshasvini), la confiance (SKOK, en Pologne, pour son produit d'assurance-épargne, à travers le sourire d'une jeune fille), la protection (Delta Life au Bangladesh, à travers les larmes d'une fille qui a pu avoir une dot grâce à l'assurance contractée par ses parents défunts) ou l'humour: le slogan «Transformer n'importe quelle veuve en bon parti» du produit prévoyance de la SINAF est devenu une référence au Brésil.

\section{Seconde nécessité: des produits relativement simples, et tenant compte de l'expertise métier des assureurs}

Le développement de la microassurance passant par la construction d'une confiance réciproque, force est de constater que la complexité nuit à la «démocratisation» de l'assurance. Aussi, face à cette situation, certains assureurs opteront pour la conception et la distribution d'un seul produit d'assurance. Ce sera souvent, pour les assureurs commerciaux, une assurance emprunteur collective et obligatoire, éventuellement avec garantie complémentaire accidentinvalidité, la plus facile à mettre en place et la plus rentable. AIG Ouganda propose ainsi, comme seul produit, une assurance emprunteur obligatoire couvrant le décès et l'accident-invalidité des clients de ses 26 IMF partenaires (24 en Ouganda, une au Malawi et une en Tanzanie). Contre le versement d'une cotisation annuelle de 87 cents, la filiale indienne d'assurance vie d'Allianz garantit pour sa part aux bénéficiaires du contrat le versement de 370 dollars en cas

28 Y. Dobel, R. Ducasse, C. Dupont, N. Khemakhem, A. Licht et F. Legrand, «La microassurance, interface entre assurance formelle et réseaux informels », projet scientifique collectif, Ecole polytechnique, 2007.

29 J.-F. Estienne, «Une autre définition de la micro-assurance est-elle possible? A la lumière de l'expérience brésilienne », intervention à la conférence «La micro-assurance: d'un monde à l'autre », Paris, Club de réflexion Haussmann, Caisse d'allocation vieillesse des agents généraux (CAVAMAC), 13 février 2007. 
de décès accidentel ou naturel de l'emprunteur d'ASA (Activists for Social Alternatives, une IMF partenaire). Le produit couvrirait, début 2007, environ 200000 personnes.

Les mutualistes privilégieront, pour diverses raisons (expertise métier, volonté de répondre au premier problème de décapitalisation des ménages, la santé), une couverture santé «universelle» et facultative, et éventuellement des produits d'assurance décès ${ }^{30}$.

Des produits prévoyance (assurances couvrant le décès, l'accident et l'invalidité d'un assuré), doublés d'une garantie santé même modeste, apparaissent cependant aujourd'hui comme une tendance de fond, révélatrice d'une recherche d'un juste milieu: la prévoyance pour sa simplicité, la couverture santé pour des raisons liées aux premiers besoins des populations.

Le développement progressif d'autres lignes de produits plus complexes (dommages, santé, voire climatiques) témoigne d'une connaissance plus soutenue et mieux maîtrisée de la microassurance.

L'assurance vie, dans sa composante épargne et non décès, suscite également un intérêt croissant parmi les microassureurs. Citons Tata-AIG en Inde, qui propose des produits d'assurance capital différé, ou SKOK en Pologne, qui distribue une assurance vie-épargne pour les membres des unions de crédit.

Cette volonté de proposer des produits d'assurance vie épargne, assortie le plus souvent d'une contre-assurance décès (versement d'un capital aux bénéficiaires du contrat en cas de décès de l'assuré avant le terme du contrat), répond en partie à un double constat: l'importance de l'épargne comme gestion du risque parmi les populations à faible revenu et la volonté de certains assureurs de canaliser cette épargne.

\section{L'assurance: un instrument de financement du développement}

\section{L'assurance et l'épargne comme outil de réduction du risque}

Le souci de l'épargne parmi les populations à faible revenu est connu. Pour les experts Marguerite Robinson et Graham Wright, l'épargne serait arrivée au premier rang des actions de la communauté de la microfinance. Jean-Michel Servet rappelle quant à lui que l'offre de possibilités d'épargne est sans nul doute dans de nombreux pays une activité plus essentielle que le crédit pour l'immense majorité des populations pauvres: "Cela explique que de nombreuses institutions de microfinance qui offrent à la fois des services d'épargne et de prêts connaissent un plus grand nombre d'épargnants que d'emprunteurs et que le volume des dépôts l'emporte sur celui des crédits. $\gg^{31}$ La volonté et la capacité d'épargner dont font preuve les populations à faible revenu dépassent clairement le cadre de la microfinance. On évoquera la diversité fonctionnelle et géographique des tontines (à tirage au sort, à enchère, chit funds en Inde, munno mukabi en Ouganda, etc.) pour de l'épargne-projet ou de l'épargne de

30 MicroInsurance Centre, Global Study on Microinsurance, 2006, consultable sur <http://www. microinsurancecentre.org $>$.

31 J.-M. Servet, op. cit., p. 258. 
précaution $^{32}$. On soulignera les motivations sociales et financières qui, loin de s'opposer, justifieront une adhésion à ces associations d'épargnants ${ }^{33}$. On rappellera que dans de nombreux pays la recherche d'une protection est l'élément qui semble le plus inciter les individus à adhérer aux tontines: «Ces résultats relèguent au second plan la nécessité d'épargner, que la littérature a toujours présentée comme facteur déterminant de l'existence des tontines. Ils montrent que les critères "paiement de l'assurance" et "régularité aux séances" sont des éléments forts constitutifs des tontines. La souscription et le versement de la prime sont généralement les premières sommes réclamées à tout nouveau membre dans plusieurs tontines visitées », souligne ainsi Sylvain Ngassam Bertelet dans un article éclairant ${ }^{34}$.

La double facette de l'épargne (sécurisation surtout et rendement dans une moindre mesure) explicite d'ailleurs un peu plus le rôle de l'assurance dans le financement du développement.

\section{L'assurance comme canal de financement long et stable}

Dans un colloque organisée conjointement par la Fédération française des sociétés d'assurances (FFSA) et l'Institut Thomas More, Gérard de La Martinière, président de la FFSA, revient notamment sur l'importance de l'assurance dans le développement économique et social d'un pays: «L'assurance favorise la croissance en mutualisant les risques liés à l'activité économique dans son ensemble, en réduisant l'incertitude des agents économiques et en canalisant un financement long et stable. ${ }^{35}$ Jean-Philippe Thierry, président-directeur général d'AGF, revient également sur le rôle moteur de l'assurance dans le développement économique et social d'une société, à travers les trois piliers suivants :

- la mutualisation des risques comme facteur stabilisateur pour la société face aux risques qui pèsent sur le développement d'une collectivité (catastrophes naturelles...) ou sur la vie d'un individu (incendie, accident...);

- la diffusion de bonnes pratiques de réduction des risques grâce à la prévention (conditionner la réduction d'une cotisation au respect par l'assuré de mesures de protection contre le risque assuré, ou accepter d'assurer un risque en contrepartie d'actions de prévention des pouvoirs publics, sont des pratiques assurantielles relativement courantes);

๑ le financement du développement économique, les assureurs étant des investisseurs à long terme (actions, obligations, immobilier) ${ }^{36}$.

32 S. Rutherford, Comment les pauvres gèrent leur argent, Paris, Groupe d'échange et de recherche technologiques (GRET); Karthala, 2002.

33 E. Baumann, «Microentreprise et gestion de la vulnérabilité en Afrique subsaharienne...», op. cit. I. Guérin, Femmes et économie solidaire, Paris, La Découverte, 2003.

34 S. Ngassam Bertelet, «A propos d'une composante négligée et oubliée: la microassurance dans l'ouest du Cameroun », Techniques financières et développement, $\mathrm{n}^{\circ}$ 78, mars 2005, pp. 116-127, citation p. 122.

35 Gérard de La Martinière, «Introduction», in Institut Thomas More, Assurance, réassurance: une autre contribution au développement. Actes du colloque du 14 juin 2005, Working Papers, no 4/FR, juillet 2006, p. 2.

36 J.-P. Thierry, site de l'AGF, <http://www.agf.fr>, page sur le développement durable. 
Et Patrick M. Liedtke, secrétaire général et directeur exécutif de l'Association de Genève, de développer ces différents éléments en soulignant le double impact positif de l'assurance sur le niveau d'épargne d'une économie: "Premièrement, elle augmente le taux d'épargne général (notamment grâce aux produits d'assurance vie), d'où des marchés plus étendus et des investissements plus importants. Deuxièmement, elle abaisse le niveau d'épargne de précaution inutile (épargne rarement investie sur les marchés de capitaux) et stimule les investissements et la consommation en réduisant le capital lié (donc improductif ou moins productif). L'assurance permet par conséquent de fournir davantage de fonds de roulement à une économie, les gens n'ayant pas à se protéger eux-mêmes contre l'éventualité d'incendie de leur résidence par exemple [...] Les mécanismes d'assurance transforment le capital dormant en capital disponible. ${ }^{37}$

Si cette transformation d'un capital dormant (Hernando de Soto parlera de capital mort) en capital productif est loin d'être aisée (faiblesse des véhicules d'investissements dans de nombreux pays) et souvent décalée quant aux origines premières de l'épargne parmi les populations à faible revenu (essentiellement de l'épargne de précaution) ${ }^{38}$, l'idée que l'assurance est une pompe à finance essentielle au développement économique et social d'un pays ne se limite pourtant pas seulement aux pays riches.

L'assureur bangladais Delta Life explicite ainsi les objectifs de ces produits de microassurance vie: "Collect small savings from the people of our country and invest the accumulated savings in profitable nation-building enterprises ${ }^{39}, \tan$ dis qu'Erard Moutassié, secrétaire général de la Conférence interafricaine des marchés d'assurance (CIMA), rappelle les origines de la création de la zone CIMA : «Il est utile de rappeler le contexte historique, économique et social qui a prévalu à la création de cette institution. A partir de 1986, la plupart de ces Etats étaient dans une situation économique très difficile, qui a d'ailleurs abouti, quelques années plus tard, en 1994, à la dévaluation de la monnaie locale (le franc CFA). La plupart de ces Etats avaient recours également aux institutions de réajustement de leur économie et au financement extérieur. Ils ont donc compris la nécessité de recourir également à l'épargne locale, l'épargne endogène. Ils ont pensé qu'il fallait assainir le secteur des assurances car il ne jouait pas le rôle qui lui était dévolu. En dehors du secteur bancaire, sur lequel jusque-là ils mettaient un accent particulier, ils ont senti la nécessité de s'intéresser à l'assurance pour mobiliser l'épargne, notamment l'épargne à long terme sur l'assurance vie. $»^{40}$

Ainsi, tant l'enjeu de mobilisation des épargnes intérieures (celles du secteur informel et semi-formel ne sont pas des moindres) que celui de réduction des vulnérabilités stimulent le développement de produits de microassurance vie

37 P.M. Liedtke, «L'assurance et son rôle prépondérant dans les économies modernes », in Institut Thomas More, op. cit., pp. 5-11, citation p. 8.

38 H. de Soto, Le mystère du capital, Paris, Flammarion, 2005. Voir également la critique nuancée de J. Sgard, «La propriété privée et les lois du capitalisme: l'approche de H. de Soto», Problèmes économiques, $\mathrm{n}^{\circ}$ 2.889, 21 décembre 2005, pp. 33-39.

39 M.J. McCord and C. Churchill (CGAP Working Group on Microinsurance), Good and Bad Pratices in Microinsurence: Delta Life, Bangladesh, Case Study, n 7, February 2005, p. 16.

40 E. Moutassié, «L'assurance dans les pays en développement: 1'exemple africain», in Institut Thomas More, op. cit., pp. 21-25, citation p. 21. 
avec contre-assurance décès. Cette assurance vie mixte permet d'éviter le piège des produits à fonds perdu, généralement rejetés par les populations. En effet, si l'assuré est en vie au terme du contrat (d'une durée de dix ans par exemple), il recevra un capital correspondant à son épargne valorisée (assurance capital différé), tandis qu'en cas de décès avant le terme du contrat, les bénéficiaires de l'assuré recevront une somme forfaitaire (temporaire décès). Delta Life (Bangladesh), Tata-AIG (Inde) ou ALMAO (Sri Lanka) proposent ce type de contrat, avec des résultats différents en termes de demande, de faisabilité et de profit.

Ces produits à durée relativement longue sont néanmoins complexes et coûteux. Ils nécessitent une grande expertise assurantielle, notamment en gestion actifpassif et en actuariat. Ils souffrent d'un risque d'inflation, entamant considérablement la valeur ajoutée de l'épargne confiée par les assurés. Enfin, la valorisation des primes (provisions techniques) en actifs financiers dépend de la performance et de la stabilité des marchés financiers, ce qui est loin d'être acquis. Ces produits nécessitent enfin un effort constant d'explications auprès des populations. Autant d'éléments qui incitent les assureurs à réduire la durée de garantie.

Ces observations ne condamnent pourtant pas les offres de produits d'assurance vie, pourvu que les assureurs répondent aux critères de facilité d'accès, de liquidité et de sécurité, déjà essentiels dans le cadre de l'épargne. Les possibilités de rachat (retrait) non pénalisantes, notamment en cas de sinistre, sont ainsi fortement recommandées.

Comprendre la gestion financière, la gestion des risques par les populations ainsi que le rôle social et économique que joue l'épargne est tout autant essentiel: il ne s'agit pas de plaquer nos propres modèles de pays fortement bancarisés et assurés sur celui d'autres populations.

D'autant que des coûts d'intermédiation généralement plus faibles, une plus grande facilité d'accès ou de retrait de son épargne, une rémunération parfois supérieure à celle proposée par les IMF formelles (quand ces dernières ont l'autorisation de collecter l'épargne), et enfin une plus grande confiance dans certaines institutions informelles (contrairement au secteur bancaire entaché par des faillites et des malversations spectaculaires) inciteraient les épargnants à se tourner plutôt vers le secteur informel ou les tontines, et à privilégier une épargne «cachée», non susceptible d'être captée par ses réseaux de dettes et d'obligations sociales.

\section{La microassurance à l'intersection du local et du global}

A ce stade, résumons-nous. L'assurance n'est pas un luxe. Elle vise à apporter des solutions concrètes en termes de gestion des risques aux populations défavorisées. La mutualisation des risques que l'assurance induit peut transcender les catégorisations parfois faciles (très pauvres/pauvres/non-pauvres), en tenant compte de l'hétérogénéité des populations et des dynamiques de pauvreté dues aux irrégularités de revenu. La diffusion de l'assurance comme réduction des risques et éventuellement comme vecteur d'investissement est cependant tributaire d'une prise en compte des cultures sociétales et d'une confiance à construire entre assurés et assureurs. 
Faite d'hybridations, de métissages et d'emprunts croisés où traditions et innovations s'enrichissent mutuellement ${ }^{41}$, la microassurance ne peut fonctionner qu'en récusant les approches conquérantes promptes à juger l'assurance informelle comme le stigmate d'une société archaïque. Son approche ne peut être que polyvalente, entre analyses sociales, culturelles, économiques, politiques, juridiques et historiques.

Pour les assurés, cela signifie que les produits aient été correctement expliqués, et leurs effets clairement démontrés (indemnisations rapides). Pour les assureurs, cela signifie acceptation de la part des assurés des règles de contrôle des fraudes, du risque moral ${ }^{42}$ et de l'antisélection ${ }^{43}$.

Entre les deux pôles, assureurs et assurés, les facilitateurs sont naturellement essentiels, tant les langages et intérêts peuvent parfois différer.

\section{Le rôle des agences de développement}

Par «facilitateurs », on entend toutes les organisations au fait des réalités quotidiennes supportées par les populations à faible revenu et susceptibles d'apporter leur expertise aux assureurs. La collaboration des ONG est souvent citée, mais les agences de développement peuvent également jouer un rôle essentiel, comme nous le rappelle Jean-Michel Debrat, de l'Agence française de développement (AFD) : «L'AFD finance de l'expertise, de la sociologie de l'organisation en espérant que dans une dizaine d'années on aura, par exemple, des organisations de producteurs qui tiendront la route... Quand on a franchi ce stade, l'assureur peut venir. Il ne faut pas faire jouer à l'assurance le rôle de développeur des mœurs. Il doit arriver au bon moment, ni trop tôt, ni trop tard, au moment où il a des clients potentiels solides et une clientèle globalement solvable. [...] Une fois le marché mûri et ces sociétés lancées, il était inutile qu'elles restent dans le giron de l'AFD. ${ }^{44}$

Ce financement d'expertise et cet apport sociologique (soutien aux structurations communautaires, mais également transfert de connaissance des cultures sociétales, des besoins et des demandes des populations) par les agences de développement sont essentiels. Ils permettent notamment aux assureurs une économie de temps et d'argent - deux éléments parfois invoqués par certains

${ }^{41}$ I. Guérin, K. Marius-Gnanou, T. Pairault et J.-M. Servet (dir.), La microfinance en Asie. Entre traditions et innovations, Paris, Karthala, 2005.

42 «Il y a risque moral lorsque la protection que fournit l'assurance encourage les individus à provoquer l'événement assuré ou à se comporter d'une manière qui augmente la probabilité qu'il survienne» («Glossaire», in C. Churchill et al., L'assurance et les institutions de microfinance, Genève, OIT, 2004, pp. 223-232, <http://www.microfinance.lu/comas/media/glossaire.pdf>). Il ne pousse pas les assurés à faire l'effort de diminuer leur niveau de risque.

Le glossaire mentionné ici donne les définitions de termes relatifs à l'assurance et à la microassurance.

43 L'antisélection émerge lorsque les assurés détiennent des informations sur le niveau de risque auxquelles les assureurs n'ont pas accès : cette asymétrie pousse les assurés à choisir un niveau de couverture adapté à leurs besoins sans que ces derniers soient connus par les assureurs. Ainsi, pour une prime donnée, seules les personnes qui présentent un niveau de risque identique ou supérieur à celui qui est associé au niveau de prime proposé vont souscrire au contrat.

44 Micro-assurance, assurance, réassurance: des outils efficaces pour le développement, entretien avec J.-M. Debrat, M. Nabeth et M. Vaté, Institut Thomas More, Notes, n 11, janvier 2007, p. 8. 
d'entre eux pour expliquer leur manque d'intérêt pour la microassurance. Aussi assistons-nous depuis quelques années à la mise en place de partenariats entre assureurs, agences de développement et institutions de microfinance ou ONG locales pour développer des programmes de microassurance.

La GTZ (coopération technique allemande) et Allianz, déjà partenaires en Inde, ont collaboré pour la conception et le développement du produit-pilote «Payung Keluarga» lancé en Indonésie. Par contre, la distribution de ce produit, l'éducation des clients à l'assurance, la collecte des primes et même le paiement des sinistres relèvent principalement des institutions de microfinance partenaires, telles que les banques rurales, les institutions villageoises de crédit, les coopératives d'épargne et de crédit et les ONG. Ces IMF agissent comme des agents pour Allianz, en raison de leur position et de leur connaissance du secteur des populations à faible revenu.

De son côté, Zurich Financial Services Group a annoncé sa nouvelle initiative de microassurance, qui constitue le premier effort concerté, à travers différentes zones d'activité du groupe, ciblant le segment des populations défavorisées; il n'a pas manqué de souligner sa collaboration avec le Bureau international du travail (BIT) et la Direction du développement et de la coopération (DDC), ces derniers s'engageant à fournir une expertise technique et à transmettre leur connaissance approfondie des communautés locales ${ }^{45}$.

Ces synergies entre assureurs, agences de développement, associations, ONG ou IMF répondent en partie aux nécessités d'articuler le local et le global, «le local permettant d'acquérir une connaissance fine des problèmes et des besoins des populations, le global permettant de peser sur les débats nationaux et la législation ${ }^{46}$, comme l'explique Isabelle Guérin. Si nous avons orienté notre article sur une vision plutôt locale, il n'est pas inutile de rappeler que la microassurance sera d'autant plus efficace qu'elle s'inscrira également dans un cadre plus macroscopique et international, lui-même respectueux de certains fondamentaux économiques et politiques.

\section{La réassurance}

La pérennité de la microassurance pose d'ailleurs très vite la question de la réassurance. Car, quelle que soit la qualité des formations d'agents et des explications fournies aux populations, l'indemnisation de tous les assurés en cas de catastrophe naturelle peut inciter l'assureur à arrêter son programme de microassurance.

VimoSEWA dut ainsi débourser 3400000 roupies (75000 dollars) à la suite du tremblement de terre au Gujarat en 2001. Cette situation incita VimoSEWA à réassurer son portefeuille, d'autant que le tremblement de terre entraîna une hausse considérable des souscriptions, le nombre d'assurés passant de 30000 à 92000. Une possibilité serait certes d'exclure les catastrophes naturelles des contrats d'assurance. Cette voie, privilégiée par certains assureurs, peut susciter

45 Zurich Financial Services Group, Zurich Increases Focus on Microinsurance, News Release, 1 February 2007.

46 I. Guérin, «Microfinance: des risques et des potentialités», in S. Allemand (dir.), La microfinance n'est plus une utopie, Paris, Autrement, 2007, p. 161. 
néanmoins une incompréhension ou un rejet des assurés au lendemain d'un sinistre majeur touchant toute la collectivité. Le secteur de la microassurance s'en trouverait discrédité. La présence de Munich Re, d'Interpolis Re et d'autres réassureurs dans la microassurance est donc une bonne nouvelle pour les promoteurs du secteur - tout comme le développement de programmes d'assurances indicielles (produits procurant une compensation pour des pertes ou manques à gagner liés aux aléas climatiques et basés sur un indice climatique $)^{47}$.

\section{La prévention des risques}

L'action des agences internationales et des gouvernements est également cruciale, notamment en matière de prévention. Michel Jarraud, secrétaire général de l'Organisation météorologique mondiale (OMM), souligne ainsi qu'un dollar investi en prévention permet d'économiser de 10 à 100 dollars de coûts de réhabilitation après des catastrophes naturelles extrêmes ${ }^{48}$. L'enjeu est essentiel, comme nous le rappelle l'économiste François-Xavier Albouy: «Si les catastrophes naturelles représentent en moyenne une somme assez faible de pertes directes qui peut être estimée à $2 \%$ du PIB mondial, les conséquences seront différentes suivant l'état de développement des économies. ${ }^{49}$ Le poids économique des catastrophes naturelles sur le Nicaragua et le Honduras fut ainsi respectivement de $50 \%$ et $37,7 \%$ du PIB, pour la seule année 1998. Cette prévention ne concerne pas que les risques de catastrophes naturelles. Le travail d'organisations sociales dans le domaine de l'éducation et de la santé est d'une telle importance que les assureurs ont tout intérêt à développer avec elles des partenariats.

\section{Le politique}

Au-delà de la réassurance, nous trouvons in fine la responsabilité de l'Etat et du politique. Nous la posons ici non pas pour aborder la possibilité de proposer, via un réassureur public, des couvertures illimitées pour des branches spécifiques telles que les catastrophes naturelles ou le terrorisme, ni pour évoquer la voie des subventions de la microassurance, à l'image des assurances agricoles, évoquées dans le premier numéro 2007 de la revue Sigma de Swiss Re. La question de l'Etat posée ici est relative à l'importance de l'Etat de droit. Aussi nécessaire soitelle, la microassurance ne peut en effet résoudre à elle seule les problèmes de la pauvreté. La présence et le partage de biens publics (éducation, santé, infrastructures collectives) influeront considérablement sur l'efficacité des programmes de microassurance. Jean-Michel Servet rappelle d'ailleurs que la pauvreté doit être

47 On lira également avec intérêt les travaux de Michel Vaté sur PlaNet Re, <http://www.institut-thomasmore.org >, p. ex. Réassurer la planète. La mondialisation financière au services des plus pauvres, Institut Thomas More, Notes, $\mathrm{n}^{\mathrm{o}}$ 1/Fr, 15 janvier 2004, et «L'objectif de sécurité alimentaire: quel rôle pour l'assurance? », in Institut Thomas More, Assurance, réassurance: une autre contribution au développement. Actes du colloque du 14 juin 2005, Working Papers, nº 4/FR, juillet 2006, pp. 44-47. Voir aussi E. de Tayo, «Planet Re: une alternative à l'aide publique au développement par l'assurance», entretien avec Michel Vaté, Afrique Expansion Mag, n² 24, 2006.

48 Y. Veyret, «Risques et prévention», Les catastrophes naturelles, dossier, Questions internationales, $\mathrm{n}^{\mathrm{o}}$ 19, mai-juin 2006, pp. 12-22, particulièrement p. 13.

49 F.-X. Albouy, «Economie des catastrophes», Les catastrophes naturelles, dossier, Questions internationales, $\mathrm{n}^{\circ} 19$, mai-juin 2006, pp. 66-72, citation p. 69. 
comprise comme la conséquence des différences hiérarchiques, autrement dit des inégalités statutaires et des discriminations qui induisent des processus de marginalisation et d'exclusion: «Plus un pays est fractionné, plus la proportion des pauvres dans la population est importante. ${ }^{50}$

Il ne s'agit pas ici de passion égalitaire, mais bel et bien de démocratie mature.

Récusant, à travers des exemples historiques, culturels ou de civilisations, l'idée que la démocratie est une construction purement occidentale, Amartya Sen conclut son essai La démocratie des autres sur la valeur universelle du système démocratique et sa fonction de formation des valeurs.

En proposant des conditions normales de conception, de tarification et de distribution qui prennent en compte la dimension individuelle, collective, solidaire, identitaire, des populations, la microassurance participe à la réflexion sur le rôle de l'assurance dans la structuration économique et sociale d'un pays, sur la qualité d'une démocratie ${ }^{51}$.

\section{Bibliographie sélective}

ADA Dialogue, $\mathrm{n}^{\circ}$ 33, 2004, thème: «La micro-assurance».

Churchill, C.F. (ed.), Protecting the Poor: A Microinsurance Compendium, Munich, Munich Re Foundation; Geneva, ILO, 2006.

Churchill, C.F., D. Liber, M.J. McCord et J. Roth, L'assurance et les institutions de microfinance, Guide technique pour le développement et la prestation de services de micro-assurance, Genève, OIT, 2004.

Nabeth, M., Micro-assurance. Défis, mise en place et commercialisation, Les Fondamentaux de l'assurance, Paris, Dalloz; L'Argus de l'Assurance, 2006.

Roth, J., M.J. McCord and D. Liber, The Landscape of Microinsurance in the World's 100 Poorest Countries, Microinsurance Centre, April 2007.

Servet, J.-M., Banquiers aux pieds nus. La microfinance, Paris, Odile Jacob, 2006.

\section{Sites Internet}

Allianz: <http://knowledge.allianz.com/en/globalissues/microfinance>.

Appui au développement autonome (ADA) : <http://www.microfinance.lu $>$.

Autorité indienne de régulation et de développement de l'Assurance : <http://www.irdaindia.org $>$.

Concertation entre les acteurs du développement des mutuelles de santé en Afrique: <http://www. concertation.org >.

Entrepreneurs de la cité : <http://www.entrepreneursdelacite.org $>$.

Groupe consultatif d'assistance aux pauvres (CGAP), Appui au développement autonome (ADA) et Groupe d'échange et de recherche technologiques (GRET) : <http://www.lamicrofinance.org $>$.

Instance de régulation et de contrôle des assureurs privés au Brésil: <http://www.susep.gov.br/menuingles/ legiseng.asp\#mi>.

Institut Thomas More, page «Aide au développement » : <http://www.institut-thomas-more.org/welcome/ developpement>.

Microinsurance Centre: $<$ http://www.microinsurancecentre.org $>$.

Munich Re: <http://www.munichre-foundation.org>.

Organisation internationale du travail (OIT):

page «Global Information on Micro-Insurance» (GIMI) : <http://www.ilo.org/gimi>;

page «Social Finance Programme», «Reducing Vulnerability»: <http://www.ilo.org/public/english/ employment/finance/vulnerab/micro/index.htm>.

Strengthening Micro Health Insurance Units for the Poor in India (consortium à but non lucratif) : $<$ http://www.microhealthinsurance-india.org $>$.

50 J.-M. Servet, op. cit., p. 463.

51 M. Nabeth, «La micro-assurance: un rôle structurant dans les nouvelles réalités sociales », Entrepreneurs de la Cité, La Lettre, nº 2, décembre 2005. 